\title{
Transmit Power Allocation for V-BLAST Systems with ZF-OSIC Detection
}

\author{
Maurizio Magarini \\ Dipartimento di Elettronica e Informazione, Politecnico di Milano \\ Piazza Leonardo da Vinci, 32, 20133 Milano, Italy \\ Email: magarini@elet.polimi.it
}

\begin{abstract}
This paper proposes a new transmit power allocation (TPA) scheme for the Vertical Bell Layered Space Time (VBLAST) system with zero-forcing ordered successive interference cancellation (ZF-OSIC) detection. The proposed TPA scheme is based on the minimization of the average probability of vector error subject to a constraint on the total average transmitted power. To derive the TPA algorithm, we consider the asymptotic approximation of the average probability of vector error that is valid at high signal-to-noise ratio. The resulting TPA scheme allocates powers to the transmit antenna elements considering very limited feedback from the receiver. Computer simulations are used to demonstrate the effectiveness of the proposed approach for a V-BLAST system with a maximum of four transmit and receive antenna elements.
\end{abstract}

\section{INTRODUCTION}

The ever increasing demand for high spectral efficiency in wireless communications has contributed to consider the introduction of architectures based on multiple antenna elements both for the transmitter and for the receiver [1]. The Vertical Bell Layered Space Time (V-BLAST) system, originally proposed in [2], is one of the most investigated architectures to achieve this higher spectral efficiency. In such a scheme the input data sequence is first demultiplexed in $N_{T}$ substreams and then transmitted, over the multiple input multiple output (MIMO) channel, through $N_{T}$ antenna elements. At the receiver, an estimate of the transmitted data symbols is produced by processing the signals received from $N_{R} \geq N_{T}$ antenna elements. The performance of the $\mathrm{V}$ BLAST architecture, in terms of error probability, is strongly dependent on the technique implemented in the receiver to detect the $N_{T}$ transmitted substreams. In this paper we focus on a V-BLAST scheme where the detection is done by zeroforcing with ordered successive interference cancellation ${ }^{1}$ (ZFOSIC) [1], [2].

In the original implementation of V-BLAST the total average transmitted power is equally divided among the transmit antenna elements. This represents the optimal solution for a VBLAST system with OSIC detection when there is no feedback from the receiver to the transmitter [3]. When feedback is present, transmit power allocation (TPA) allows us to obtain a performance improvement [4]. The TPA problem has been investigated in [4], [5], [6]. All such approaches consider,

\footnotetext{
${ }^{1} \mathrm{~A}$ better performance is obtained with minimum-mean square error OSIC detection. Since its analytical performance evaluation is complicated we do not consider it in the present work.
}

as a performance criterion to be optimized, the instantaneous probability of symbol or vector error subject to a constraint on the total average transmitted power. The main difficulty in dealing with the constrained minimization of instantaneous probabilities of error is the presence in their analytical expression of the complementary cumulative distribution function of the Gaussian noise ( $Q$-function). For such schemes the optimization problem has been dealt with either by resorting to non-linear optimization methods [5] or approximating the $Q$-function as proposed in [4], [6].

In this paper we propose a new TPA scheme based on the minimization of the average probability of vector error subject to a constraint on the total average transmitted power. In particular, we consider its asymptotic approximation at high signalto-noise ratio (SNR). The resulting TPA scheme assigns power to each transmit antenna considering very limited feedback from the receiver that consists of the average SNR and the order in which the substreams are detected. This introduces a further simplification compared to [4], [5], [6] where the information sent by the receiver consists of the power to be transmitted from each antenna according to the given channel realization.

The paper is organized as follows. The model of the system we focus on is given in section II. The proposed TPA scheme is introduced in section III. Experimental results are shown in section IV and, finally, conclusions are drawn in section V.

\section{System Model}

In this paper, we only consider a V-BLAST scheme with equal numbers of transmit and receive antenna elements, i.e. $N_{R}=N_{T}=N$. Let a denote the $N \times 1$ vector of independent transmitted symbols each having the same modulation order and unitary average power. The received signal vector at a particular time instant is represented in complex baseband form as

$$
\mathbf{r}=\mathbf{H} \mathbf{P}^{1 / 2} \mathbf{a}+\mathbf{w}
$$

where $\mathbf{w}$ is the $N \times 1$ noise vector of i.i.d. complex Gaussian random variables (RVs) with zero mean and variance $\sigma_{w}^{2}$, $\mathbf{H}$ is the $N \times N$ MIMO channel matrix whose entries are i.i.d. RVs having uniform-distributed phase and Rayleighdistributed magnitude with average power equal to 1 and $\mathbf{P}=$ $\operatorname{diag}\left[p_{1}, \ldots, p_{N}\right]$ is a diagonal matrix whose $n$-th entry $p_{n}$ denotes the average power transmitted from the $n$-th antenna. 
The value of $p_{n}$ is established at the transmitter on the basis of the feedback sent by the receiver. The constraint $\operatorname{trace}(\mathbf{P})=1$ is set on the total average transmitted power. We assume that both $\mathbf{H}$ and $\sigma_{w}^{2}$ are perfectly estimated at the receiver and that the feedback channel is error free. The average SNR is defined as $\bar{\gamma} \triangleq 1 / \sigma_{w}^{2}$.

As in [4], [5], [6], we assume that the ZF-OSIC detection process implemented at the receiver coincides with that of the conventional V-BLAST, that is, based on the ordered postdetection SNR obtained when $\mathbf{P}=\frac{1}{N} \mathbf{I}_{N}$ ( $\mathbf{I}_{N}$ denotes the $N \times N$ identity matrix). Therefore, the detection order is not influenced by the TPA scheme.

\section{The Proposed TPA Scheme}

In absence of error propagation the ZF-OSIC detection process decouples the channel matrix into a set of independent parallel channels with increasing diversity order, being $i$ the diversity order at the $i$-th processing step for a system with equal number of transmit and receive antenna elements [1]. According to this view, we can apply all the modulation and/or power adaptation strategies that have been devised to optimize different performance criteria in case of transmission over independent parallel channels where channel state information is available at the transmitter (see [7] and references therein). In particular, we are interested in the optimization of the vector error rate for a fixed data rate and a given total average transmitted power. For such a case, the optimal solution is given in [8] and consists in an algorithm that assigns the modulation order and the power on each channel so that the probabilities of symbol error are equalized on all the parallel channels. However, for the scheme under investigation we have the additional constraint that the same modulation order must be used on all the parallel channels and, therefore, a TPA strategy equalizing the probabilities of symbol error does not provide the optimal solution. A better performance is obtained if one considers a TPA strategy that minimizes the average probability of vector error.

The average probability of vector error in a V-BLAST system with ZF-OSIC detection is upperbounded as [9]

$$
P_{v}\left(\bar{\gamma}_{c, 1}, \cdots, \bar{\gamma}_{c, N}\right) \leq \sum_{i=1}^{N} P_{e, i}\left(\bar{\gamma}_{c, i}\right),
$$

where $P_{e, i}\left(\bar{\gamma}_{c, i}\right)$ denotes the average conditional probability of symbol error at $i$-th detection step as a function of the average SNR per channel $\bar{\gamma}_{c, i}$ given that no error has occurred in the previous step. Actually, only $P_{e, i}\left(\bar{\gamma}_{c, i}\right), i=2, \ldots, N$, represent average conditional probabilities of symbol error while $P_{e, 1}\left(\bar{\gamma}_{c, 1}\right)$ cannot be affected by the error propagation in the "previous step". The average SNR per channel at $i$-th step is defined as

$$
\bar{\gamma}_{c, i}\left(\alpha_{i}\right) \triangleq \alpha_{i} / \sigma_{w}^{2}, \quad i=1, \ldots, N,
$$

where $\alpha_{i}$ denotes the average transmitted power allocated to the transmitted substream detected in such a step. Average powers are assigned to transmit antenna elements according to the detection ordering communicated by the receiver, that is $p_{k_{i}}=\alpha_{i}$, where $k_{i} \in\{1, \ldots, N\}$ represents the integer identifying the transmitted substream to be detected at the $i$ th step. Note that equation (2) also provides an upper bound to the average probability of symbol error with error propagation [9]. Our TPA scheme is based on the minimization of (2) subject to the constraint on the total average transmitted power. The cost function is written as

$$
J\left(\alpha_{1}, \cdots, \alpha_{N}, \lambda\right) \leq \sum_{i=1}^{N} P_{e, i}\left(\alpha_{i}\right)+\lambda\left(\sum_{i=1}^{N} \alpha_{i}-1\right),
$$

where $\lambda$ is the Lagrange multiplier. Note that in (4) the dependence of $P_{e, i}$ on $\alpha_{i}$ has been made explicit by using (3). The minimization of (4) gives the optimal powers $\alpha_{i}$ for a given average SNR.

In the following the optimization problem is considered separately for the cases $N=2,3,4$ and, for the sake of simplicity, we refer to coherent BPSK.

\section{A. $2 \times 2$ case}

In this case the optimization problem is solved by minimizing the average probability of vector error given in (2). By taking into account the total average transmitted power constraint, we respectively denote as $\alpha$ and $1-\alpha$ the average powers assigned to the first and the second detected substreams. The average SNRs per channel at the first and second steps are respectively

$$
\bar{\gamma}_{c, 1}(\alpha) \triangleq \frac{\alpha}{\sigma_{w}^{2}}, \quad \bar{\gamma}_{c, 2}(\alpha) \triangleq \frac{1-\alpha}{\sigma_{w}^{2}} .
$$

According to the SNRs per channel defined above and considering the analysis developed in [3], [9], it is possible to write the exact average probabilities of symbol error at the first and second steps. Their use in (2) would lead to an average probability of vector error that does not lend itself to a manageable form from an optimization point of view. For this reason we consider their asymptotic approximations that are valid at high SNR

$$
P_{e, 1}(\alpha) \approx \frac{1}{8 \bar{\gamma}_{c, 1}(\alpha)}, \quad P_{e, 2}(\alpha) \approx \frac{3}{8 \bar{\gamma}_{c, 2}^{2}(\alpha)} .
$$

The asymptotic approximation of the average probability of vector error as a function of $\alpha$ is

$$
P_{v}(\alpha) \approx \frac{\sigma_{w}^{2}}{8 \alpha}+\frac{3 \sigma_{w}^{4}}{8(1-\alpha)^{2}}
$$

By setting $d P_{v}(\alpha) / d \alpha=0$ and solving the resulting third degree equation we obtain the value of $\bar{\alpha}$ that minimizes (7) for the given $\sigma_{w}^{2}$. The dependence of the solution from $\sigma_{w}^{2}$ is denoted as $\bar{\alpha}(\bar{\gamma})$. We assign the average powers $\bar{\alpha}(\bar{\gamma})$ and $1-$ $\bar{\alpha}(\bar{\gamma})$ respectively to the transmit antenna elements from which the first and second detected substreams are sent according to the value of $\bar{\gamma}$ and the detection order communicated by the receiver. We emphasize that the optimal powers are not calculated at the receiver. Their values are established at the transmitter on the basis of the average SNR and then assigned to the transmit antenna elements on the basis of the detection order. 


\section{B. $3 \times 3$ case}

The exact analytical expressions of the average probabilities of symbol error at the different detection steps are known only for the case of $N_{T}=2$ and arbitrary $N_{R}$ [3], [9]. In order to derive their analytic asymptotic approximations for an $N \times N$ V-BLAST system with $N>2$, we extend the analysis developed in [3], [9] for the case of two transmit antenna elements by taking into account the conjectures used in [10] to analyze the asymptotic approximation of the outage probability for $N=3,4$. The outage probability is defined as the probability that the output signal level falls below a prescribed value. The asymptotic approximations of the average probabilities of symbol error can be easily computed from the asymptotic approximations of the outage probabilities as shown in [9].

The optimal ordering procedure at the first detection step for an $N \times N$ system results in an SNR gain of $N$ in the asymptotic approximation of the outage probability compared to the asymptotic behavior of the Rayleigh distribution [10]. The corresponding asymptotic approximation of the average probability of symbol error is [11]

$$
P_{e, 1}\left(\alpha_{1}\right) \approx \frac{1}{4 N \bar{\gamma}_{c, 1}\left(\alpha_{1}\right)} .
$$

To derive the asymptotic approximations of the average probabilities of symbol error at the second and third steps we adopt the solution proposed in [10]. According to this procedure, the outage probabilities at the second and third steps for a $3 \times 3$ scheme are respectively considered the same of those at the first and second steps of a $2 \times 3$ system. However, this assumption can be considered true only if the channel statistics for both the second and third steps is not affected by the optimal ordering in the first detection step. As shown in [10], by comparing the analytical results to Monte Carlo (MC) simulations, the asymptotic approximation of the outage probability at the second step for $N=3$ is overestimated by $3 \mathrm{~dB}$. It is also shown that the outage probability at the second step is predicted extremely well by the second order maximum ratio combining curve. According to this observation and taking into account the analysis developed in [3], the asymptotic approximation of the average probability of symbol error at the second step is

$$
P_{e, 2}\left(\alpha_{2}\right) \approx \frac{9}{64 \bar{\gamma}_{c, 2}^{2}\left(\alpha_{2}\right)} .
$$

As far as the outage probability at the third step is concerned, it is shown in [10] that there is a good agreement between the analysis and MC simulations. The expression for the asymptotic approximation of the average probability of symbol error at the third step is

$$
P_{e, 3}\left(\alpha_{3}\right) \approx \frac{15}{32 \bar{\gamma}_{c, 3}^{3}\left(\alpha_{3}\right)} .
$$

MC simulations have been carried out to evaluate the validity of the asymptotic approximations derived in (8), (9) and (10).
The average powers to be transmitted for a given SNR are obtained by minimizing (4) after substitution of the asymptotic approximations given in (8), (9) and (10). As in [5], to find the minimum of (4) we have used the MATLAB function FMINCON. This function attempts to find the constrained minimum of a scalar function of several variables starting at an initial estimate. As in the previous case, the average powers are allocated to different transmit antenna elements on the basis of the average SNR and the detection order communicated by the receiver.

\section{C. $4 \times 4$ case}

Also for this case the asymptotic approximation of the average probability of symbol error at the first step is obtained from (8). To compute $P_{e, 2}\left(\alpha_{2}\right)$ we use the asymptotic approximation of the outage probability at the first step of a $3 \times 4$ system given in [10]. A good approximation of the asymptotic average probability of symbol error is

$$
P_{e, 2}\left(\alpha_{2}\right) \approx \frac{3}{32 \bar{\gamma}_{c, 2}^{2}\left(\alpha_{2}\right)} .
$$

To obtain (11) we have introduced a multiplicative factor in order to compensate the performance difference between the analytical curve of the outage probability and MC simulations shown in [10]. By using the same procedure adopted for the case $N=3$ with exactly the same considerations, it is possible to derive the asymptotic approximations of the average probabilities of symbol error at the third and fourth steps. They are respectively obtained by extending the asymptotic approximations of the average probabilities of symbol error at the first and second steps of a $2 \times 4$ system, and are given by

$$
P_{e, 3}\left(\alpha_{3}\right) \approx \frac{1}{8 \bar{\gamma}_{c, 3}^{3}\left(\alpha_{3}\right)}
$$

and

$$
P_{e, 4}\left(\alpha_{4}\right) \approx \frac{35}{64 \bar{\gamma}_{c, 4}^{4}\left(\alpha_{4}\right)} .
$$

The procedure to determine and assign the optimal powers at the transmitter for $N=4$ is the same of that we have illustrated in subsection III-B for $N=3$.

\section{Simulation RESUlts}

Fig. 1 reports the performance comparison between our TPA scheme and the conventional one for the $2 \times 2$ case. For such a situation we have found that the asymptotic approximation of the average probability of vector error provides a good estimate of the true performance in the average SNR region defined by $\bar{\gamma} \geq 15 \mathrm{~dB}$. The figure shows that at $10^{-3}$ the proposed TPA scheme allows us to obtain a gain of $1 \mathrm{~dB}$ over the conventional one. In order to validate the asymptotic approximation, numerical results obtained by MC simulations are also reported in Fig. 1. We observe that no difference exists between the asymptotic approximation and MC simulations. Fig. 2 reports the conditional average probabilities of symbol error at the first and second detection steps for the proposed TPA scheme and the conventional one in the $2 \times 2$ case. 


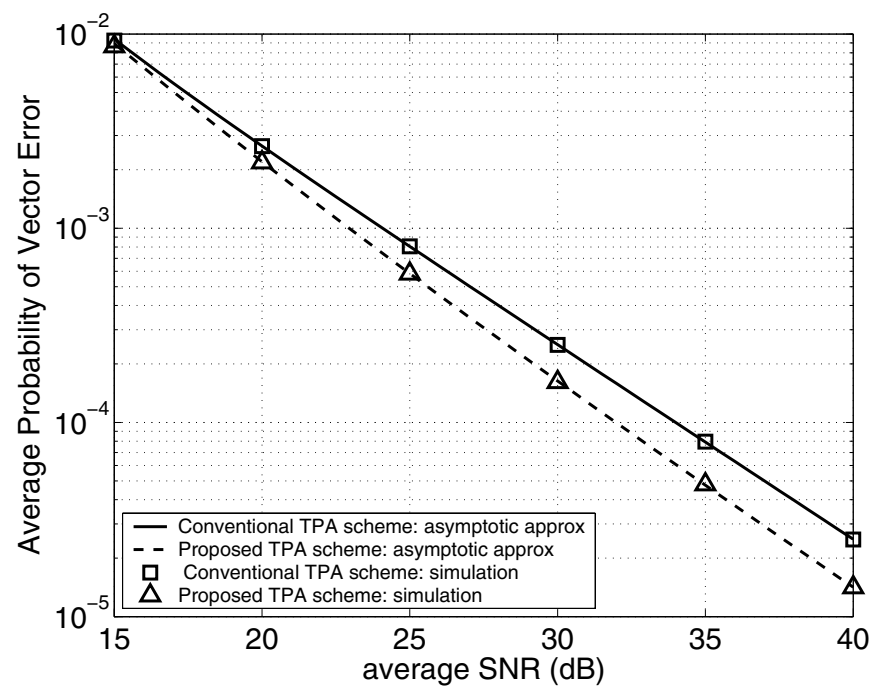

Fig. 1. Average probability of vector error for a $2 \times 2$ V-BLAST system with ZF-OSIC.

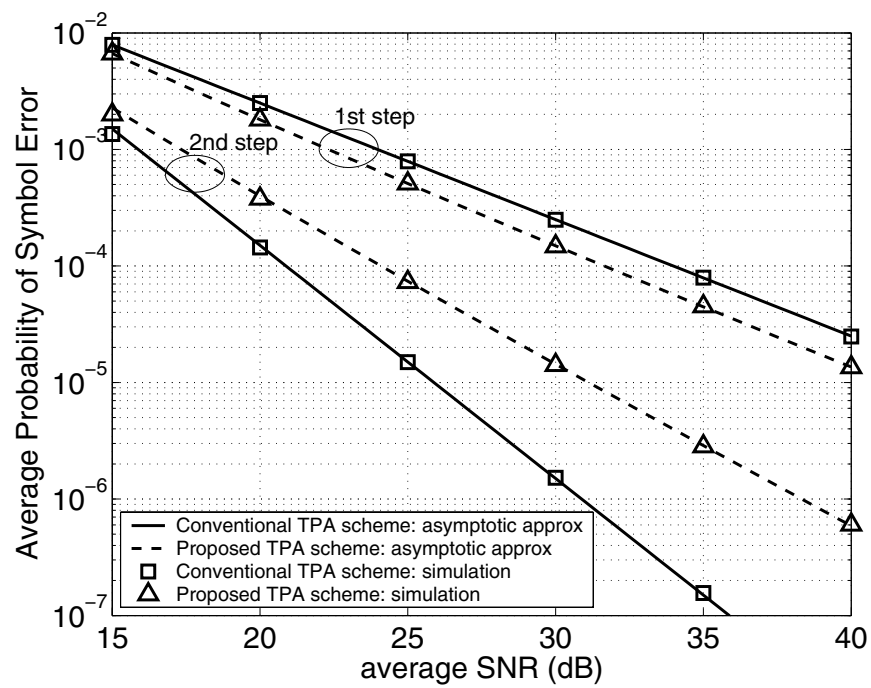

Fig. 2. Average probabilities of symbol error at the first and second detection steps for a $2 \times 2$ V-BLAST system with ZF-OSIC.

As the figure shows, there is a good agreement between the asymptotic approximations of the average probabilities of symbol error and MC simulations. For both the cases we observe that the average probability of vector error is dominated by the probability of symbol error at the first step. Fig. 3 reports the average powers allocated to the transmit antenna elements from which the first and second detected substreams are transmitted. Note that as the average SNR increases the average power $p_{k_{1}}$ tends asymptotically to 1 . Since the average probability of vector error is dominated by the average probability of symbol error at the first step, we conclude that for $N=2$ the asymptotic gain of the proposed TPA scheme over the conventional one is $3 \mathrm{~dB}$ (the conventional TPA scheme allocates the average power $1 / 2$ to both the two transmit antenna elements). Numerical results for

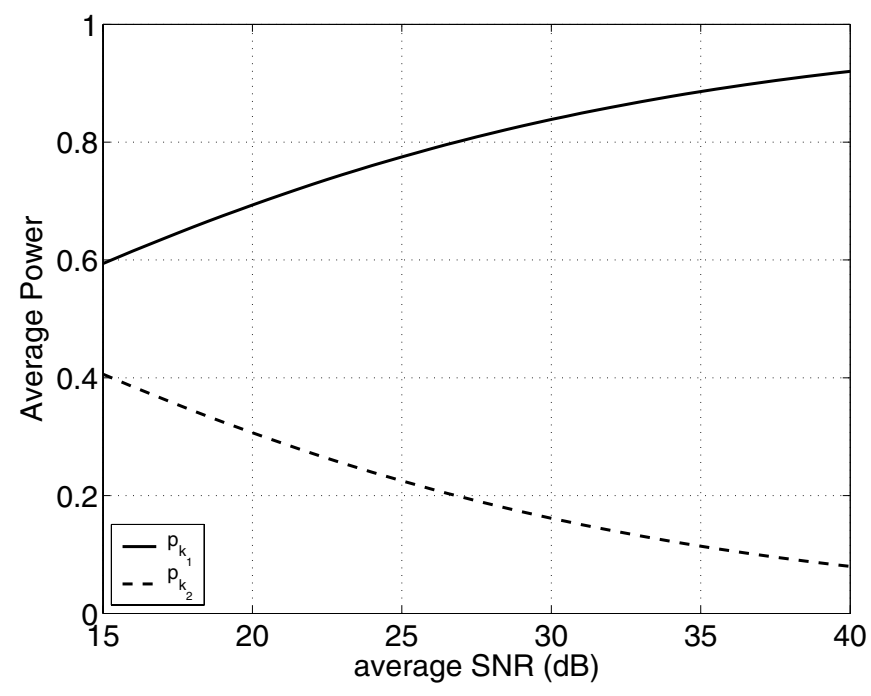

Fig. 3. Average power allocation at the first and second detection steps for a $2 \times 2$ system.

the $3 \times 3$ and the $4 \times 4$ cases are reported respectively in figures 4 and 5 . At $10^{-3}$ the proposed TPA scheme gives a gain of 2 $\mathrm{dB}$ and of $3 \mathrm{~dB}$ respectively for $N=3$ and $N=4$ compared to the conventional one. Also for this case no differences have been observed between the asymptotic approximations and MC simulations in the region $\bar{\gamma} \geq 15 \mathrm{~dB}$. The superior gains obtained for $N=3,4$ compared to $N=2$ can be easily explained by observing that asymptotically the total average transmitted power is allocated to the antenna element from which the first detected substream is transmitted. In the case $N=4$, for example, this means a factor 4 of gain over the conventional TPA scheme, that is, an asymptotic gain of $6 \mathrm{~dB}$. For all the examples we emphasize that in the region $\bar{\gamma}<15 \mathrm{~dB}$ the asymptotic approximation does not provide a good estimate of the true performance. In this region the proposed TPA scheme would not provide a gain compared to the conventional one due to the lack of validity of the asymptotic approximations.

\section{CONCLUSIONS}

This paper presents a new TPA scheme for V-BLAST systems with ZF-OSIC detection based on the minimization of the asymptotic approximation of the average probability of vector error. The power allocation at the transmitter is done on the basis of very limited feedback from the receiver that consists only in the average SNR and the detection order. Simulation results have been used to demonstrate the effectiveness of the proposed TPA approach compared to the conventional equal power allocation scheme for $N \times N$ systems, with $N=2,3,4$.

\section{REFERENCES}

[1] A. Paulraj, R. Nabar, D. Gore, "Introduction to space-time wireless communications," Cambridge, UK: Cambridge University Press, 2003. 


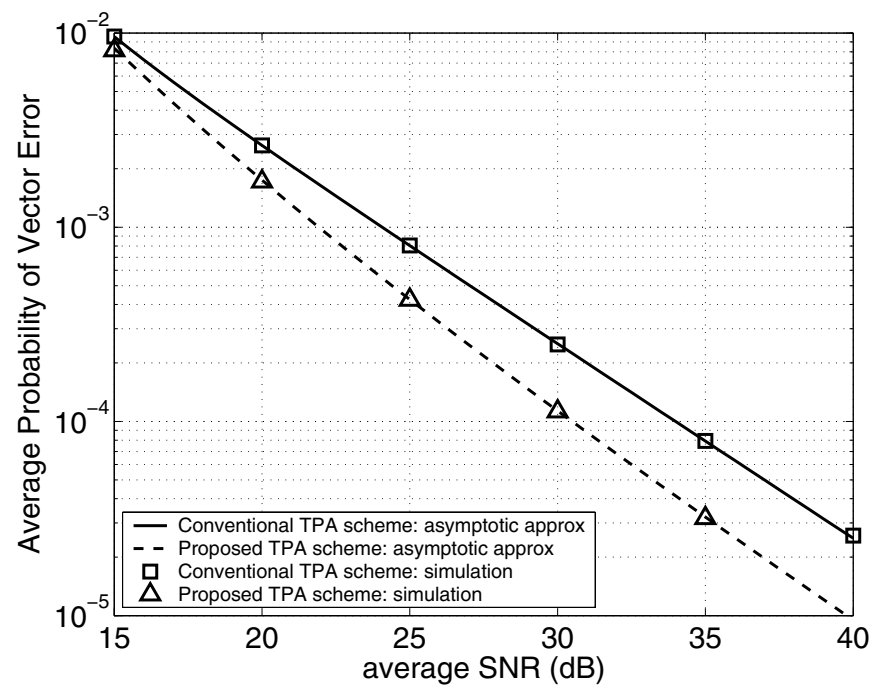

Fig. 4. Average probability of vector error for a $3 \times 3$ V-BLAST system with ZF-OSIC.

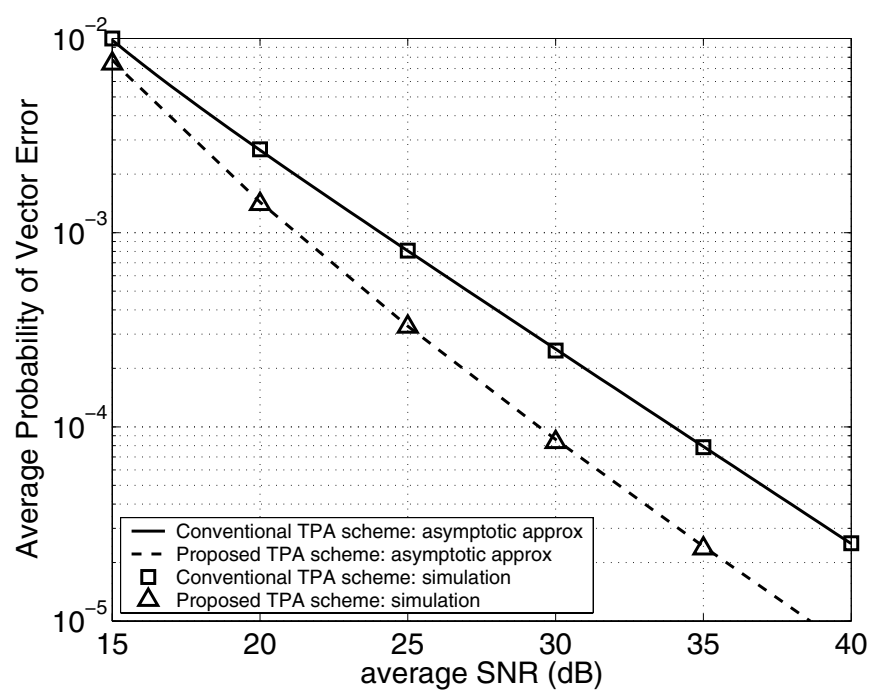

Fig. 5. Average probability of vector error for a $4 \times 4$ V-BLAST system with ZF-OSIC.

[2] P. W. Wolniansky, G. J. Foschini, G. D. Golden, R. A. Valenzuela, "VBLAST: an architecture for realizing very high data rates over the richscattering wireless channel," in Proc. ISSSE, Pisa, Italy, pp. 295-300, 29 Sept.-2 Oct. 1998

[3] R. T. Xu, C. M. Lau, "Analytical approach of V-BLAST performance with two transmit antennas," in Proc. WCNC, New Orleans, LA, pp. 396-401, Mar. 2005.

[4] S. H. Nam, Oh-S. Shin, K. B. Lee, "Transmit power allocation for a modified V-BLAST system," IEEE Trans. Commun., vol. 52, pp. 10741079, July 2004.

[5] R. Kalbasi, D. D. Falconer, A. H. Banihashemi, "Optimum power allocation for a V-BLAST system with two antennas at the transmitter," IEEE Commun. Lett., vol. 9, pp. 826-828, Sept. 2005.

[6] N. Wang, S. D. Blostein,"Minimum BER power allocation for MIMO spatial multiplexing systems," in Proc. ICC, Seoul, Korea, pp. 22822286, May 2005.

[7] T. Hunziker, D. Dahlhaus, "Optimal power allocation for OFDM systems with ideal bit-interleaving and hard decision decoding," in Proc. ICC, Anchorage, AK, pp. 3392-3397, May 2003.
[8] R. F. H. Fischer, J. B. Huber, "A new loading algorithm for discrete multitone transmission," in Proc. Globecom, London, UK, pp. 724-728, Nov. 1996.

[9] S. Loyka, F. Gagnon, "Performance analysis of the V-BLAST algorithm: an analytical approach," IEEE Trans. Wireless Commun., vol. 3, pp. 1326-1337, July 2004.

[10] S. Loyka, F. Gagnon, "Analytical framework for outage and BER analysis of the V-BLAST algorithm," in Proc. IZS, Zurich, Switzerland, pp. 120-123, Feb. 2004.

[11] J. G. Proakis "Digital communications (4th edition)," New York, NY McGraw-Hill, 2001. 